\title{
Principal Flow for Tubular Objects with Non-Circular Cross-sections
}

\begin{abstract}
Various anatomical objects are tubular in shape. These structures can be modeled by describing their curvilinear path and the cross-sectional shape along the path. However, most research on tubular object segmentation has focused on vascular systems, and often assumes a circular cross-section. These techniques are not readily applicable to anatomy such as the cochlea, which has a non-circular cross-sectional shape. We present the Principal Flow Filter, which calculates the local flow vector along a tubular object with a non-circular cross-section. It can be used to extract the centerline orientation and thus track along the tube, and as a basis for segmentation. We present results from generated data with a variety of cross-sectional shapes. The filter is shown to rapidly and robustly converge to the true orientation. We also analyse a CT scan of a human cochlea, with promising results.
\end{abstract}

\section{Introduction}

A variety of anatomical structures are tubular in shape; for example, the vascular system, the bronchi, the colon and the cochlea. We are interested in modeling the cochlea and analysing its shape, for which we need to track the centerline and extract the cross-sections from Computed Tomography (CT) data. The human cochlea resembles a shell, while its cross-section resembles a cardioid (a rounded "B") that varies along its length. A model that captures crosssectional shape could be used in diagnosis by identifying abnormalities, and also in surgery training and planning.

Several techniques exist for segmenting tubular objects from volumetric data. Most have focused on vascular networks, since these represent the most common tubular structures in the body. However, most of these approaches assume a circular or elliptical cross-section, and many assume a constant cross-sectional radius. This is inadequate to represent significant detail in many organs, where crosssectional shape can have great clinical significance.

We have developed the Principal Flow Filter to explicitly deal with non-circular cross-sections. It calculates the flow vector along a tube, and can be used to extract the centerline, and thus track along the tube for segmentation.

This paper first discusses existing approaches to tubular object segmentation. Then we present the Principal Flow Filter and its design. We show the results from several generated data sets, as well as from a CT scan. Finally we discuss how this filter can be used for full tubular object segmentation.

\section{Background}

The intrinsic shape characteristics of a tubular object can be described by two components: the centerline path and the cross-section along the path. Binford [2] first proposed the Generalised Cylinder (GC), a spatial curve defining the path of the object, and a cross-section (typically circular or elliptical) that can vary as a function of the path length. Zerroug and Nevatia [10] extended the GC model, describing the Right Generalised Cylinder (RGC) which constrains the cross-section to be orthogonal to the tangent of the path. We employ the RGC model for this research.

Wang et al. describe a local intensity tracking algorithm to extract the path of a cochlear implant electrode in vivo [8]. The implant is metallic with a highly regular shape, and appears with a circular cross-section in CT images. It can easily be segmented by thresholding, since the intensity of the electrode is far greater than the surrounding bone and tissue. Principal Components Analysis (PCA) is applied to a local region, and the approximate centerline is tracked along the eigenvector with the largest eigenvalue. The orthogonal planes are extracted and the center of mass used to calculate the next center point.

Flasque et al. present a technique for extracting vascular networks from pre-segmented 3D images [4]. The algorithm extracts the centerline of the vasculature using a moving parallelepiped that tracks along the target tube. The representation is based on a RGC, and uses an interpolated Bspline for the centerline path, with cross-sections extracted orthogonal to the centerline path. It appears that the center of mass determines the centerpoint.

The two approaches above do not directly deal with the problem of segmentation. A more complex approach is taken by Aylward and Bullitt [1], who describe a technique 
for extracting the centerline of the vascular system. An intensity ridge is obtained by treating an $\mathrm{N}$-dimensional image as a surface in N+1-dimensional space. The surface is a height field, mapping image intensity to height. The algorithm climbs image gradients and follows ridges along the length of the tubular object, using a local Hessian to direct the normal plane. It assumes the tube has a circular cross-section, and explicitly tests that the aspect ratio of the cross-section is not too elliptical. The local tubular radius is incorporated into the representation.

A novel approach was introduced by Frangi et al. [5] for the analysis of vascular structures in MRA data. The centerline is first extracted using a deformable model or snake [6], then the vessel wall is extracted. A multi-scale filter based on the local Hessian is used to drive the attracting force toward the intensity ridge of the centerline, and a local discriminant function that enhances tubular profiles is used to guide tracking. A B-spline models the centerline of the tube, and a deformable surface models the vessel wall. The surface is initialised with a circular cross-section and then deformed to fit the wall.

Yim et al. [9], present an analysis of and extensions to Frangi's tubular co-ordinate system. However this approach requires the user to manually specify points to form the centerline, and so does not address centerline extraction.

Krissian presents a multi-scale approach to vascular segmentation [7]. This also employs a RGC model, and uses the eigenvectors of the local Hessian to guide the extraction.

Nearly all the approaches described above utilise a local Hessian to guide tracking along the tube by following the principal eigenvector. This presupposes that the tube exhibits a Gaussian intensity profile with a local maximum at its center (such as described in [5]); ie. a nearly circular cross-section. Objects with non-circular cross-sections do not have a Gaussian intensity profile with a single local maximum at the center, hence these approaches are not well suited to this class of problem.

\section{Method}

Consider a volumetric data set of a tubular object. At a given point $P$, we wish to obtain the local flow orientation $V$ (ie. the tangent to the implicit centerline). The image gradient will be strongest along the tube walls, normal to the tube surface (Figure 1a). If we consider a sufficiently narrow region of the tube (Figure $1 b$ ), the vectors will tend to be approximately coplanar, oriented toward the center. If we take a large number of cross-products of pairs of gradient vectors (Figure 1c), we can compute the mean orientation and thus the local flow vector. Given this flow vector, we can compute the orthogonal cross-sectional plane and then the centerline, leading to an iterative segmentation that tracks along the tube. Considering only the tube walls ob-

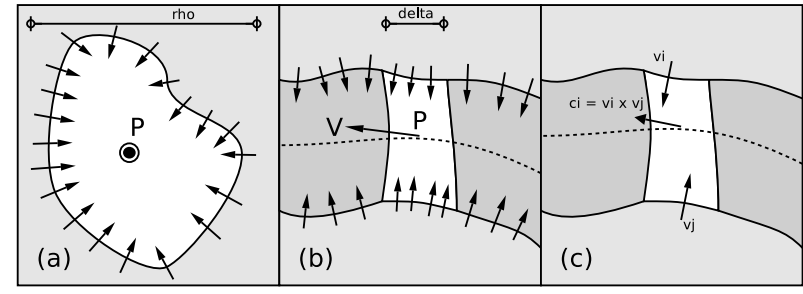

Figure 1. Tubular object: (a) centerpoint of cross section, (b) narrow section of tube with flow vector, (c) pair of gradient vectors and their cross-product

viates the need for a local intensity maxixmum along the centerline, and thus non-circular cross-sections can be processed.

Let $I$ be the original volumetric image. The algorithm is manually initialised by specifying a starting point $P_{0}$ inside the tube, and orientation $V_{0}$, pointing approximately along the tube. This defines the initial Volume of Interest (VOI) $R$, of dimensions $\rho \times \rho \times \delta$, with $P$ at its center and oriented such that $V$ defines the new $\mathrm{Z}$-axis. We apply the Laplacian operator $\nabla$ to obtain the image gradient $\nabla R$ in the resampled volume. This gradient will be strong along the walls, and the gradient vectors will generally be oriented in approximately the same plane, pointing toward the center of the tube. We randomly sample $N$ gradient vectors $v_{n}$ from $\nabla R$, thresholding the gradient magnitude (above some $\epsilon$ ) to reject noise:

$$
\mathcal{V}=\left\{v: v_{i}=S(\nabla R), i \in[0, N],\left\|v_{i}\right\|>\epsilon\right\}
$$

where $S$ is a pseudo-random sampling function. We then take the vector cross-product of all pairs from $\mathcal{V}$ :

$$
\mathcal{C}=\left\{c: c_{i}=v_{m} \times v_{n}, \forall m, n \in[0, M], m \neq n\right\}
$$

The average orientation of this set $\mathcal{C}$ of cross-products will point along the tube. We project these flow vectors into a 2D space $\Theta$ using the angles of $c$ in the $X Z$ and $Y Z$ planes (see for example Figure 4):

$\Theta=\left\{\left(t_{x z}, t_{y z}\right): \begin{array}{l}t_{x z}=\arctan \left(c_{x}, c_{z}\right) \\ t_{y z}=\arctan \left(c_{y}, c_{z}\right)\end{array}\right\}, t \in \mathcal{R}^{2}$

A cluster in this plot indicates strong support for the average orientation. However, minor variations in the shape, combined with sampling artifacts and other noise sources, introduce a fairly high degree of noise. We employ the RANSAC algorithm [3] to robustly calculate the flow vector $c_{n}$ with the greatest support from $\mathcal{C}$. The support for 
each flow vector is calculated using a Euclidean distance function in $\Theta$. The mean orientation is calculated from the support set, which is projected back into the original coordinate frame of the volume $I$ to give $v^{\prime}$. The Principal Flow Vector is thus given by:

$$
\mathcal{F}_{t}=\frac{\mathcal{F}_{t-1}+v^{\prime}}{\left\|\mathcal{F}_{t-1}+v^{\prime}\right\|}
$$

The parameter selection for $\rho$ is determined by the data acquisition parameters and the expected maximum crosssectional width of the tube. The $\delta$ parameter should be selected as inversely proportional to the maximum curvature of the tube; empirical results suggest a good range is between 5 and 9.

\section{Results}

First, volumetric test data was generated at a resolution of $50^{3}$ voxels, containing tubes with a variety of crosssectional shapes: circular, a figure- 8 and a clover-leaf shape (see Figure 2). The Flow Filter was initialised with the point $P$ approximately in the center of the tube, and the orientation $V$ approximately $34^{\circ}$ deviated from the known true orientation. The algorithm was run for 20 iterations each. Figure 3 shows the filter converging to known the true orientation in less than 10 iterations for each case. The calculated Principal Flow Vector is within $1^{\circ}$ of true (circular: $0.75^{\circ}$, eight: $0.33^{\circ}$, clover: $0.69^{\circ}$ ).

The 2D projection of the flow vectors is shown in Figure 4 for the clover data set, for iterations 1 and 20. In the first iteration, there is a certain amount of noise, but still a strong cluster just left and below center $(0,0)$. By the last iteration, there is very little dispersion as the Principal Flow Vector is almost directly aligned along the tube.

Tests were also carried out on a CT scan $(512 \times 512 \times$ 114) of a human temporal bone. The algorithm was initialised inside the basal turn of the right cochlea, and run for 100 iterations. The calculated flow vector is shown in Figure 5 superimposed over front and side projections of the cochlea CT data. The algorithm converged quickly, and oscillated about a band of $1^{\circ}$ (as can be seen in Figure 7). In the absence of ground truth from this clinical data, visual inspection indicates the result closely matches the expected orientation. Full medical validation will be performed in the next series of tests.

\section{Conclusion}

We have presented a novel approach to calculating tubular flow, a critical step enabling automated segmentation of tubular objects. The approach has been demonstrated on test data with a variety of cross-sectional shapes, as well
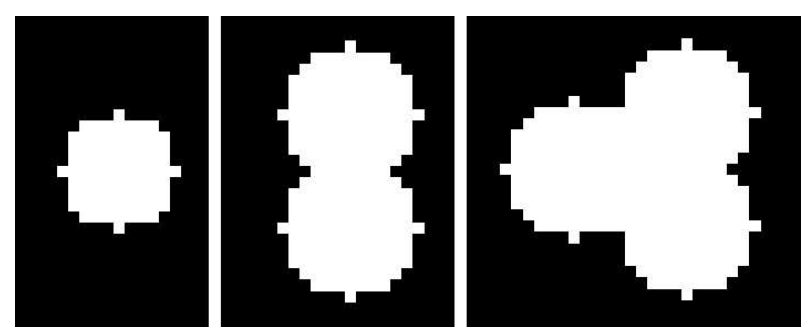

Figure 2. Volumetric test data was generated (at low resolution) with (a) circular, (b) figure8 and (c) clover-leaf cross-sections.

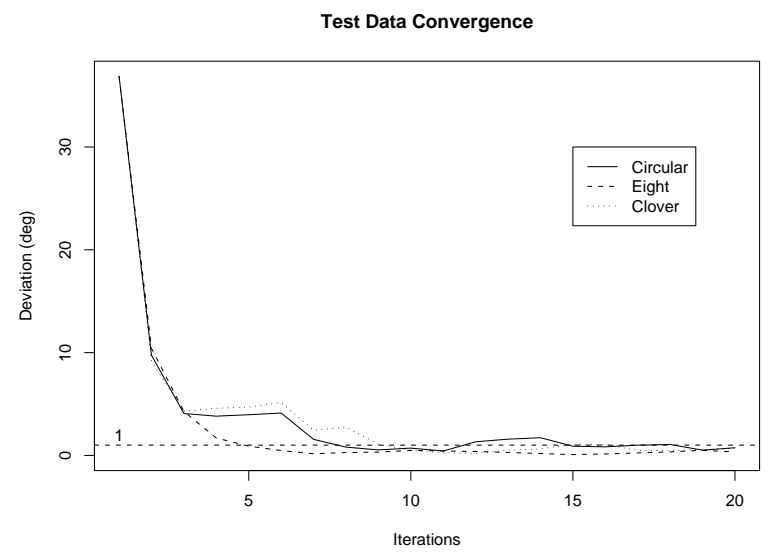

Figure 3. The flow vector converges to true for three different shaped cross-sections.
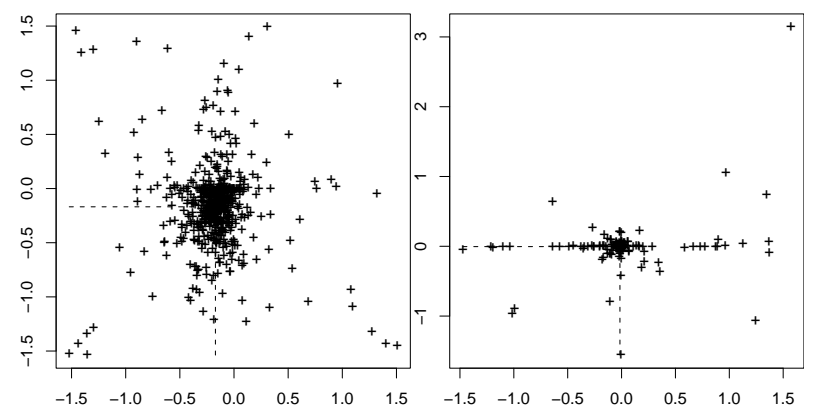

Figure 4. Flow vectors from the clover test data projected into 2D space, after the (a) first and (b) the last (20th) iteration. The dashed lines show the calculated mean orientation in each axis. 


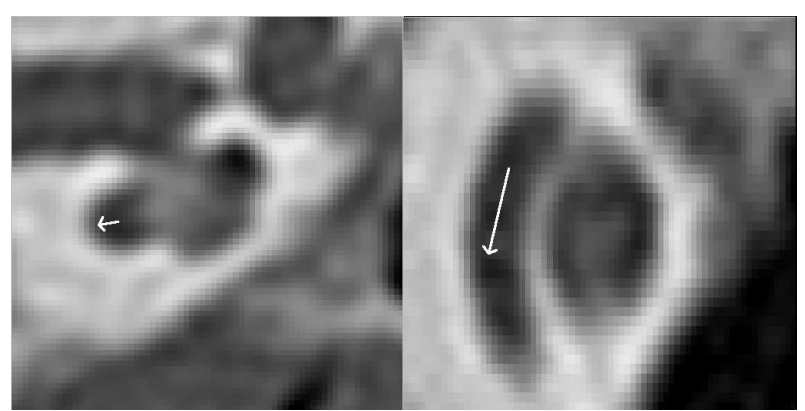

Figure 5. Front and side projection of human cochlea, showing calculated flow vector.



Figure 6. Projection plots for cochlea CT, over several iterations.

Cochlea Convergence

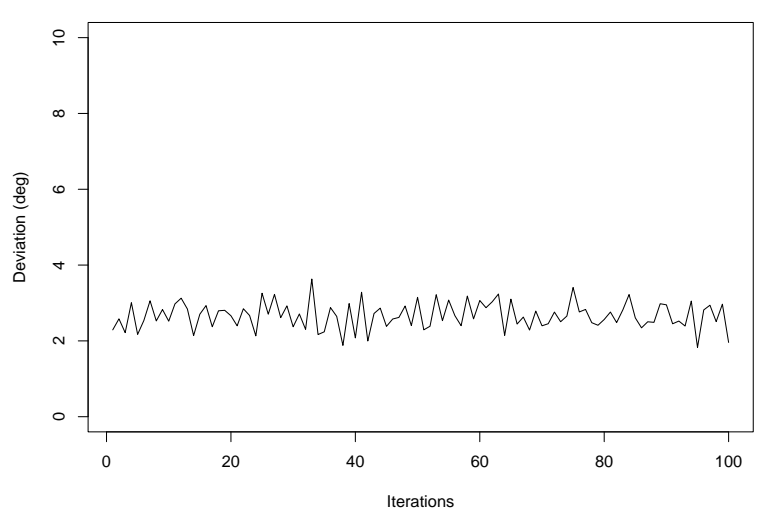

Figure 7. Convergence to reference with Cochlea CT data: mean jitter is $<1^{\circ}$. as on a section of a clinlcal CT data. It has proven robust with respect to initialisation, recovering from a significant starting divergence. It can robustly handle unusual crosssections, such as may appear in anatomy. Encouraging results were also shown from the human cochlea.

This technique enables the full-scale tracking and extraction of tubular objects. Given the Principal Flow Vector, we can extract the orthogonal plane and derive the centerline from the center of mass of the tube. The shape of the crosssections along the tube can be analysed to detect abnormalities, and also to build an atlas of shape variation. Ongoing research is using this approach to track along and fully segment the human cochlea.

Thanks to Dr Stephen O'Leary, the Department of Otolaryngology at The University of Melbourne, and the Radiology unit at the Royal Victorian Eye and Ear Hospital.

\section{References}

[1] S. Aylward and E. Bullitt. Initialization, noise, singularities, and scale in height ridge traversal for tubular object centerline extraction. IEEE Transactions on Medical Imaging, 21(2):61-75, February 2002.

[2] T. Binford. Visual perception by computer. In IEEE Conference on Systems Science and Cybernetics, 1971.

[3] M. A. Fischler and R. C. Bolles. Random sample consensus: a paradigm for model fi tting with applications to image analysis and automated cartography. Communications of the ACM, 24(6):381-395, 1981.

[4] N. Flasque, M. Desvignes, J. M. Constans, and M. Revenu. Vessel surface reconstruction with a tubular deformable model. In 4th IEEE Southwest Symposium on Image Analysis and Interpretation, pages 96-100. IEEE, April 2000.

[5] A. Frangi, W. Niessen, R. Hoogeveen, T. van Walsum, and M. Viergever. Model-based quantitation of 3-D magnetic resonance angiographic images. IEEE Transactions on Medical Imaging, 18(10):946-956, October 1999.

[6] M. Kass, A. Witkin, and D. Terzopolous. Snakes: Active contour models. International Journal of Computer Vision, 1:321-331, 1988.

[7] K. Krissian, G. M. R. Vaillant, Y. Trousset, and N. Ayache. Model-based multiscale detection of 3D vessels. In Computer Vision and Pattern Recognition, pages 722-727. IEEE, June 1998.

[8] G. Wang, M. Vannier, M. Skinner, W. Kalender, A. Polacin, and D. Ketten. Unwrapping cochlear implants by spiral ct. IEEE Transactions on Biomedical Engineering, 43(9):891900, September 1996.

[9] P. Yim, J. Cebral, R. Mullick, H. Marcos, and P. Choyke. Vessel surface reconstruction with a tubular deformable model. IEEE Transactions on Medical Imaging, 20(12):1411-1421, December 2001.

[10] M. Zerroug and R. Nevatia. Three-dimensional descriptions based on the analysis of the invariant and quasi-invariant properties of some curved-axis generalized cylinders. IEEE Transactions on Pattern Analysis and Machine Intelligence, 18(3):237-253, March 1996. 